This is an electronic reprint of the original article. This reprint may differ from the original in pagination and typographic detail.

Author(s): Gao, Ying; Cronin, Neil; Pesola, Arto; Finni Juutinen, Taija

Title: $\quad$ Muscle activity patterns and spinal shrinkage in office workers using a sit-stand workstation versus a sit workstation

Year: $\quad 2016$

Version:

Please cite the original version:

Gao, Y., Cronin, N., Pesola, A., \& Finni Juutinen, T. (2016). Muscle activity patterns and spinal shrinkage in office workers using a sit-stand workstation versus a sit workstation. Ergonomics, 59(10), 1267-1274.

https://doi.org/10.1080/00140139.2016.1139750

All material supplied via JYX is protected by copyright and other intellectual property rights, and duplication or sale of all or part of any of the repository collections is not permitted, except that material may be duplicated by you for your research use or educational purposes in electronic or print form. You must obtain permission for any other use. Electronic or print copies may not be offered, whether for sale or otherwise to anyone who is not an authorised user. 


\section{Muscle Activity Patterns and Spinal Shrinkage in Office Workers Using a Sit-Stand Workstation versus a Sit Workstation}

Ying Gao $^{1 *}$, Neil J. Cronin ${ }^{1}$, Arto J. Pesola ${ }^{1}$, Taija Finni ${ }^{1}$

${ }^{1}$ Neuromuscular Research Center, Department of Biology of Physical Activity, University of Jyväskylä, P.O.Box 35, FI-40014,Jyväskylä, Finland

*Corresponding author

Email addresses:

YG: yigao@jyu.fi

NJC: neil.j.cronin@jyu.fi

AJP: arto.j.pesola@jyu.fi

TF: taija.finni@jyu.fi

\section{Acknowledgements}

Ying Gao was supported by the China Scholarship Council (NO.201206320092), China. International Society of Biomechanics is acknowledged for the Congress Travel Grant (\#5439). The authors declare that they have no competing interests. 


\begin{abstract}
Reducing sitting time by means of sit-stand workstations is an emerging trend but further evidence is needed regarding their health benefits. This cross-sectional study compared work time muscle activity patterns and spinal shrinkage between office workers (aged 24-62, 58.3\% female) who used either a sit-stand workstation (Sit-Stand group, $\mathrm{n}=10$ ) or a traditional sit workstation (Sit group, $\mathrm{n}=14$ ) for at least the past three months. During one typical workday, muscle inactivity and activity from quadriceps and hamstrings were monitored using electromyography (EMG) shorts, and spinal shrinkage was measured using stadiometry before and after the workday. Compared with the Sit group, the Sit-Stand group had less muscle inactivity time $(66.2 \pm 17.1 \%$ vs. $80.9 \pm 6.4 \%, \mathrm{p}=0.014)$ and more light muscle activity time $(26.1 \pm 12.3 \%$ vs. $14.9 \pm 6.3 \%, p=0.019)$ with no significant difference in spinal shrinkage $(5.62 \pm 2.75 \mathrm{~mm}$ vs. $6.11 \pm 2.44 \mathrm{~mm})$. This study provides evidence that working with sit-stand workstations can promote more light muscle activity time and less inactivity without negative effects on spinal shrinkage.
\end{abstract}




\section{Keywords}

sit-stand workstation, spinal shrinkage, muscle inactivity and muscle activity, office workers 


\section{Practitioner Summary}

This cross-sectional study compared the effects of using a sit-stand workstation to a sit workstation on muscle activity patterns and spinal shrinkage in office workers. It provides evidence that working with a sit-stand workstation can promote more light muscle activity time and less inactivity without negative effects on spinal shrinkage. 


\section{Introduction}

Office work exposes people to high amounts of sedentary time, consisting of long periods of sitting with minimal muscle activity (Pesola et al. 2014). Too much sitting is adversely associated with cardio-metabolic (Wilmot et al. 2012) and increased risks of musculoskeletal symptoms (Lis et al. 2007). To reduce these risks in office workers, reducing sitting time is emerging as a novel and compelling workplace-health priority (Chau et al. 2010; Healy et al. 2012).

With the development of ergonomic office furniture, sit-stand workstations have been reported to reduce self-reported (Pronk et al. 2012) and objectively measured sitting time at work (Dutta et al. 2014; Chau et al. 2014), which is predominantly replaced by standing (Alkhajah et al. 2012; Healy et al. 2013; Straker et al. 2013). Although sitting and standing both involve low levels of energy expenditure, standing requires more muscle activity in the lower extremities than sitting (Hamilton et al. 2007). It has been shown that even short bouts of light muscle activity can improve cardio-metabolic risk factors (Dunstan et al. 2012; Duvivier et al. 2013). Greater muscle inactivity time has also been shown to be adversely associated with several cardio-metabolic biomarkers in healthy office workers (Pesola et al. 2015), suggesting a potential effect of sit-stand workstations on metabolic health. Thus, further evidence is required concerning muscle inactivity and activity patterns comparing office workers using sit-stand or sit workstations.

The use of sit-stand workstations has also been linked with improved musculoskeletal health (Robertson et al. 2013), but current evidence of such benefits is mainly based on perceived discomfort (Karakolis and Callaghan 2014). Stadiometry is a reliable, valid and objective method that has been used to quantify the height loss of intervertebral discs resulting from spinal shrinkage (Lewis and Fowler 2009). Spinal shrinkage is an indicator of disc compression, and activities with greater compressive loads increase spinal shrinkage (Eklund and Corlett 1984; Kingma et al. 2000). Several studies have compared the effects of trunk movement and postural changes on spinal shrinkage in work situations (Paul and Helander 1995; Van Dieën, De Looze and Hermans 2001; Van Dieën and Oude Vrielink 1998), and standing has been shown to cause more spinal shrinkage (Van Deursen et al. 2005) or less recovery of shrinkage than sitting (Beynon and Reilly 2001). Although there is a lack of normative data on typical workday spinal shrinkage, standing is associated with increased spinal shrinkage (Igic, Ryser, and Elfering 2013), and spinal shrinkage is greater at work performed in a standing compared to a sitting position (Leivseth and Drerup 1997). Thus, suggestions to encourage more standing at work should be made with caution, and the effects of using sit-stand workstations on musculoskeletal outcomes should be investigated with objective measures.

A recent Cochrane review concluded that sit-stand workstations provide health benefits compared to sit workstations at work, but that the quality of the studies is low (Shrestha et al. 2014). In order to understand the potential health related outcomes when using sit-stand workstations, the purpose of this cross-sectional study was to compare the activity of lower extremity muscles and spinal shrinkage between office workers who use either a sit-stand 
workstation or a traditional sit workstation. Quadriceps and hamstring muscle inactivity and activity time and spinal shrinkage were assessed during one typical workday in an ecologically valid setting.

\section{Methods}

\subsection{Participants and design}

The study was carried out at the University of Jyväskylä, Finland, in the Faculty of Sport and Health Sciences. Participants were recruited using advertisements distributed via university web pages. In total, 34 of the 122 individuals who responded agreed to participate in the experimental study. They met the inclusion criteria of being full-time office employees, healthy and non-pregnant. Exclusion criteria were self-reported chronic, long-term musculoskeletal disease or progressive neurological disease (1 excluded). From the remaining 33 participants, sufficient data were obtained during one workday from 24 participants (aged 24-62, 58.3\% female). Reasons for exclusion due to insufficient data were missing data $(n=4)$, electromyography $(E M G)$ contained prolonged $(>30 \mathrm{~min})$ artifacts in all 4 channels $(\mathrm{n}=2)$ and/or stature was outside of the $158-191 \mathrm{~cm}$ range measurable by the stadiometer $(\mathrm{n}=3)$. Of the final sample, 10 participants reported working with a sit-stand workstation (Sit-Stand group) and 14 participants used a traditional sit workstation (Sit group). All participants had used these workstations for at least the past three months. The Sit-Stand group had electronically adjustable sit-stand workstations (ISKU, Finland) that allow workers to select a sitting or standing position at work. This cross-sectional study was performed in a real work environment and participants were measured during one typical workday whilst performing normal computer tasks. No monetary incentive was offered to the participants. The study received ethical approval from the Ethics Committee of the University of Jyväskylä, and all participants provided written informed consent prior to participation.

\subsection{Protocol}

Before the measurements, demographic data were collected using an online questionnaire. Participants attended the laboratory where they were given instructions and anthropometric assessments were done. Waist-to-hip circumference (WHR) and body mass (InBody 720, Biospace Ltd, Seoul, Korea) were measured before lunch and body mass index (BMI, kg/m2) was calculated using height (with accuracy of $0.1 \mathrm{~cm}$ ) and weight (with accuracy of $0.1 \mathrm{~kg}$ ). Participants were instructed not to participate in any strenuous physical activity for $24 \mathrm{~h}$ prior to the measurement day. In the morning of the measurements, the participants arrived at the laboratory before work for baseline assessments. EMG shorts (Myontec Ltd, Kuopio) were put on and set to record, after which the participants performed the following tasks while quadriceps and hamstring muscle EMG were measured: lying down, sitting, standing, leaning on right/left hip, squatting with body weight and normal walking. Stature was measured using a stadiometer. Before the measurements, participants remained standing for 2 minutes to minimize heel pad shrinking (Foreman and Linge 1989). Reproducibility and possible 
measurement error were controlled by a sufficient familiarization session, where standard deviation was less than $0.5 \mathrm{~mm}$ for consecutive measurements (Rodackiet al. 2001). During the measurement participants kept their arms crossed over their chests, and several breathing cycles were allowed in a comfortable standing posture. Repeated measurements were performed over 30s each, and participants stepped off the stadiometer between measurements. After the laboratory assessments, the participants began their normal workday while wearing the EMG shorts, and they kept a diary during work hours. At the end of the workday, a work routine diary was completed and perceived musculoskeletal discomfort in different body parts was rated. The stature measurements were repeated when the participants returned to the laboratory for post assessments.

\subsection{Questionnaire and diary}

The questionnaire was implemented electronically using MrInterview (SPSS DimensionNet, version 5.5). This system is based on the E-mail distribution of a link to the actual survey and participants reply via a web browser on the Internet. Participants gave demographic information including gender, age, education, computer use at work, physical activity, workstation type, occupational sedentary time and perceived work ability (Tuomi et al. 1998). Sedentary time in the workplace was assessed with the following questions: "How much of your entire workday, on average, did you sit/stand during the last 3 months? (0-100\%)", "At your workstation, how much time during your computer work, on average, did you spend sitting/standing during the last 3 months? (0-100\%)", and "What is the average amount of time you spend a day sitting during your free time (excluding work time)? (0-8 hours)". Participants also answered the question "How would you rate your current work ability compared with lifetime best", on a scale from 0 (completely unable to work) to 10 (work ability at its best).

Participants recorded their daily routines (i.e. lunch, time away from office, toilet visit) in a diary. At the end of the workday, participants rated perceived musculoskeletal discomfort in different body parts (neck and shoulders, upper limbs, back and lower limbs) on a 5-point scale ranging from 1 (no discomfort) to 5 (strong discomfort).

\subsection{EMG-derived muscle activity}

Muscle activity was assessed using textile EMG electrodes embedded into shorts made of knitted fabric similar to elastic clothes (Myontec Ltd, Kuopio). Signals were stored in a small waist-mounted module (Finni et al. 2007). Electrodes were positioned in a bipolar configuration over the muscle bellies of the left and right quadriceps and hamstring muscles. These EMG shorts have been tested previously for validity, repeatability and feasibility, and a detailed description of the recording devices has been reported (Finni et al. 2007). Adequate repeatability and consistency of EMG signals, both day-to-day and within-day, have been tested in our laboratory and reported in previous studies (Tikkanen et al, 2013; Pesola et al, 2014).

The EMG signals from quadriceps and hamstring muscles were normalized channel by channel to EMG values measured during submaximal walking. Averaged EMG data were 
used from 10 consecutive step cycles during overground walking along a 30m walkway measured at individually preferred speed $(4.1 \pm 0.4 \mathrm{~km} / \mathrm{h})$. In order to reflect the overall inactivity and activity periods, normalized data were averaged to produce mean thigh muscle EMG. The inactivity threshold was set individually at $90 \%$ of the mean EMG amplitude measured during quiet standing for 10s, in accordance with our previous studies (Tikkanen et al. 2013; Pesola et al. 2014). The threshold for light and moderate-to-vigorous intensity was defined individually as the mean EMG value during normal walking. Mean EMG amplitudes from the reference tests and thresholds for different activity levels are shown in Figure 1.

Occasional artifacts (e.g. toilet visits) were manually removed from every channel. If an EMG artifact was longer than 30 minutes, the particular channel was removed from the analysis. In total, $2.5 \%$ of recording time was visually considered to be artifact and removed from 78 channels of 24 participants. Signals were corrected for possible baseline drift using a moving 5 minute window (Tikkanen et al. 2013). The normalized, averaged, artifact-free EMG data were further processed using a custom-made Matlab script (MATLAB, MathWorks, Massachusetts). Based on the thresholds, signals were analyzed for total muscle inactivity duration ( $\mathrm{min}$ ), total muscle activity duration ( $\mathrm{min}$ ), light and moderate-to-vigorous muscle activity duration (min), duration of the five longest continuous inactivity periods (min), and number of activity bursts throughout the workday, as described previously (Tikkanen et al. 2013; Pesola et al. 2014).

\subsection{Spinal shrinkage}

Stadiometry is a reliable and valid method of assessing spinal shrinkage (Lewis and Fowler 2009). The stadiometer used in this study (University of Jyväskylä, Finland) was designed to measure stature with $0.01 \mathrm{~mm}$ accuracy (measuring range of 158 to $191 \mathrm{~cm}$ ) in a standing posture whilst participants leaned on a frame tilted back $15^{\circ}$ from the vertical (Rodacki et al. 2001). Individually preset posture of the spine at levels of C4 (cervical lordosis curve), T8 (thoracic kyphosis) and L3 (lumbar lordosis curve) with adjustable support enabled precise control of the depth of each spinal contour. Stature was taken as the average of two 30s measurements sampled at $20 \mathrm{~Hz}$. Pre-post work time stature loss was calculated and used to reflect spinal shrinkage during the workday (Leivseth and Drerup 1997). In our study, the average standard deviation of the repeated stature measurements was $0.19 \pm 0.08 \mathrm{~mm}$ for before work measures and $0.21 \pm 0.11 \mathrm{~mm}$ for after work measures. Thus, the repeatability of our measures was considered acceptable ( $\mathrm{SD}<0.5 \mathrm{~mm}$ ) (Rodacki et al. 2001; Healey et al. 2005).

\subsection{Statistical analysis}

Means and standard deviations were used for descriptive evaluation of the data. The statistical significance of group-specific characteristics was tested with independent t-test (normally distributed data) or Mann-Whitney U (non-normally distributed data) for continuous variables, and chi-square test or chi-square test with Fisher's exact test for categorical variables. Statistical analyses were conducted using IBM SPSS for Windows 22.0 (SPSS Inc., Chicago, IL, USA) with a significance level of $\mathrm{p}<0.05$ (two-tailed). 


\section{Results}

Demographics and self-reported occupational sedentary time of the study groups are presented in Table 1. There were no significant differences between groups in age, height, body mass, BMI, WHR, gender distribution, computer use at work or physical activity level. The age range of participants was 24-62 and most of them had university level education $(95.8 \%)$. The majority of participants met the minimum recommendations for participation in physical activity (Haskell et al. 2007). Compared with the Sit group, participants from the Sit-Stand group reported less sitting time $(62.0 \pm 13.0 \%$ vs. $83.6 \pm 12.0 \%, \mathrm{p}=0.001)$ and computer sitting time $(68.5 \pm 22.1 \%$ vs. $87.1 \pm 17.7 \%, \mathrm{p}=0.028)$ during the workday, as well as more standing time $(36.5 \pm 21.9 \%$ vs. $18.2 \pm 21.8 \%, \mathrm{p}=0.031)$ and computer standing time $(23.7 \pm 18.0 \%$ vs. $3.0 \pm 10.7 \%, \mathrm{p}<0.001)$. Average daily leisure time sitting was $3.5 \pm 1.2$ hours and did not differ between groups. There was no significant difference in perceived work ability between groups ( $8.7 \pm 1.1$ and $8.8 \pm 0.9$, respectively).

Muscle activity and spinal shrinkage data for both groups are presented in Table 2. Averaged EMG recording time of all participants was $403.9 \pm 47.0$ minutes and did not significantly differ between groups. Compared with the Sit group, the Sit-Stand group exhibited less muscle inactivity time $(66.2 \pm 17.1 \%$ vs. $80.9 \pm 6.4 \%, \mathrm{p}=0.014)$ and more light muscle activity time $(26.1 \pm 12.3 \%$ vs. $14.9 \pm 6.3 \%, p=0.019)$ at work. There were no significant differences in moderate-to-vigorous muscle activity time, total number of bursts or the sum of the 5 longest muscle inactivity periods between groups. Furthermore, both groups exhibited spinal shrinkage but there was no significant difference between groups (Table 2). At the end of the measurement day, the Sit-Stand group perceived less musculoskeletal discomfort in the back than the Sit group $(1.4 \pm 0.7$ vs. $2.4 \pm 1.1, \mathrm{p}=0.020)$. In neck and shoulders, upper limbs and lower limbs, there were no group differences in musculoskeletal discomfort.

\section{Discussion}

The results of this cross-sectional study showed that compared with office workers using traditional sit workstations, those using sit-stand workstations exhibited less muscle inactivity time and more light muscle activity time, with less perceived musculoskeletal discomfort in the back, and without negative effects on spinal shrinkage.

\subsection{EMG-derived muscle activity}

In normal daily life the thigh muscles are inactive for over $65 \%$ of the day (Tikkanen et al. 2013) and over $78 \%$ of work time, even in physically active office workers (Pesola et al. 2014). In the current study, the activity patterns of quadriceps and hamstring muscles showed $15 \%$ less inactivity and $11 \%$ more light activity in workers using sit-stand workstations compared to users of traditional sit workstations. This is in line with previous reports, which found that the introduction of sit-stand workstations was associated with reductions in sitting 
time of 5\%-26\% of work time (Alkhajah et al. 2012; Chau et al. 2014; Dutta et al. 2014; Pronk et al. 2012; Straker et al. 2013). Although energy expenditure when using a sit-stand workstation increases by $1.2 \mathrm{kcal} / \mathrm{min}$ compared with a traditional sit workstation (TudorLocke et al. 2013), less muscle inactivity, even by the small magnitude of $15 \%$, may provide positive signals for metabolic health (Pesola et al. 2015). It has been reported that a difference of $2 \mathrm{~h} 18 \mathrm{~min}$ in daily muscle inactivity is beneficially associated with metabolic markers such as fasting HDL cholesterol and triglycerides independent of moderate-tovigorous muscle activity time (Pesola et al. 2015).

While muscle inactivity time was reduced in the Sit-Stand group, there were no significant differences in moderate-to-vigorous muscle activity time between groups. This may reflect the sedentary nature of office work, whereby moderate-to-vigorous activity occupies only a fraction of daily work time, and lower intensity physical activity predominates (Clemes, O'Connell and Edwardson 2014). Interestingly, although the Sit-Stand group exhibited less total muscle inactivity time, neither the sum of the 5 longest inactivity periods nor the total number of bursts differed between groups. Similar results were also found in intervention studies (Alkhajah et al. 2012; Healy et al. 2013) and a cross-sectional study (Straker et al. 2013) that examined reductions in sitting time, but these studies did not detect changes in accumulated sitting bouts, stepping time or number of sit-stand transitions. Although there was no significant difference in the sum of the 5 longest inactivity periods, the almost 20 minute difference between groups may have been sufficient to provide metabolic health benefits, but this needs to be confirmed in future studies. Because sitting periods accrued in bouts of 30 minutes or more are related to health risks, regular postural changes are recommended even for workers who use sit-stand workstations (Healy et al. 2012). Experimental studies have shown that frequent bouts of activity which reduce total sedentary time have a positive effect on metabolic variables (Duvivier et al. 2013; Dunstan et al. 2012), but it is still unclear whether the total or uninterrupted sitting time is important. Thus in the present study the lack of difference in total number of muscle activity bursts may only imply that the pattern of postural transitions was similar between groups. Due to greater total duration of muscle activity, the use of a sit-stand workstation likely benefits metabolic outcomes even though it may not affect the number of activity bursts.

\subsection{Spinal shrinkage}

Spinal shrinkage has been used in ergonomic evaluations of working situations to reflect the effects of spinal loading, which can be assumed to have predictive value regarding health effects (Igic, Ryser, and Elfering 2013; Paul and Helander 1995; Van Dieën and Oude Vrielink 1998). In this study we used perceived musculoskeletal discomfort and spinal shrinkage as indicators of potential effects. The Sit-Stand group reported less discomfort in the back, which corresponds well with review results concluding that sit-stand workstations are effective at reducing local discomfort in the back (Karakolis and Callaghan 2014). In the current study, the mean spinal shrinkage was $5.91 \mathrm{~mm}(0.35 \%$ of stature $)$ after a typical office workday. This is comparable with results from another study that reported mean spinal shrinkage of $0.45 \%$ of stature at the end of a bout of standing work, with no significant differences between different work-rest schedules (Van Dieën and Oude Vrielink 1998). A 
study focusing on work involving low physical activity reported shrinkage of the thoracolumbar spine of $4.75 \mathrm{~mm}$ or $6.95 \mathrm{~mm}$, after $6.5 \mathrm{~h}$ of work in a prolonged sitting or standing posture, respectively. Corresponding values in the lumbar spine were $1.73 \mathrm{~mm}$ or $4.16 \mathrm{~mm}$ (Leivseth and Drerup 1997). However, in view of task specificity and large interindividual variability in spinal shrinkage, comparisons across different groups and studies are difficult. Interestingly, we found no statistically significant difference in spinal shrinkage between groups. Although static standing has been reported to result in greater spinal shrinkage than static sitting (Van Deursen et al. 2005), in dynamic situations, where office workers change positions with higher job control (Igic, Ryser, and Elfering 2013), it may be possible to mitigate increases in spinal shrinkage. In addition, using a sit-stand workstation may involve body movement which results in reduced spinal shrinkage or enhanced recovery of shrinkage (Van Dieën, De Looze and Hermans 2001). Importantly, the range of spinal shrinkage $(1.55 \mathrm{~mm}-10.55 \mathrm{~mm})$ in both groups of the present study was below the highest permissible spinal shrinkage of $21 \mathrm{~mm}$ in order to prevent occupational low back pain (Ismaila and Charles-Owaba 2008). Thus this study provides evidence from an occupational ergonomic perspective that habitual use of a sit-stand workstation does not cause negative effects on spinal shrinkage. However, it should be highlighted that excessive standing time at work may not be healthy (Igic, Ryser, and Elfering 2013). The effect of prolonged standing on spinal health, over periods exceeding the habitual times measured in the present study, should be examined to be able to recommend safe limits for workday standing time to sit-stand workstation users.

\subsection{Strengths and limitations}

Beyond simply reporting differences in sitting time, the current study measured muscle activity and spinal shrinkage with objective measurements, and compared office workers who either worked with a sit-stand workstation or traditional sit workstation in a real workplace. This study is one of very few that have measured muscle activity using EMG shorts, which have been reported to provide an accurate and detailed assessment of muscle activity across the entire continuum of daily life (Tikkanen et al. 2013). Compared with analysis of activity outcomes from accelerometers and postural data (Chastin and Granat 2010), EMG as a direct measure of muscle activity provides further physiological insight into changes in muscle activity and inactivity time, and the associated cardio-metabolic impact when reducing sitting time (Tikkanen et al. 2014; Pesola et al. 2014, 2015).

There are several limitations of the current study. This cross-sectional study included data from only one workday, which could make the effects of day-to-day variation and result in potential bias. To counteract this limitation, the participants were asked to choose a typical workday representative of their habitual daily work (Baranowski et al. 2008) for the day of measurements. Although several studies have explored the optimal sit-stand ratio regarding health outcomes (Van Dieën and Oude Vrielink 1998; Paul and Helander 1995), we did not

give any instructions regarding the sit-stand ratio but asked them to behave as they would in a typical workday. Regarding some outcomes, the statistical power might have been too low to detect significant differences. For example, the difference in the sum of the 5 longest inactivity periods was almost 20 minutes, but it was not statistically significant between 
groups. For future studies, 17 participants in both groups would be required to achieve more than $90 \%$ power for the primary outcome of muscle inactivity time. Larger-scale randomizedcontrolled interventions with follow-up assessments are needed to investigate the potential causal long-term impact of sit-stand workstations on various health outcomes.

\section{Conclusion}

This cross-sectional study showed that office workers using sit-stand workstations had $\sim 15 \%$ less muscle inactivity time and $\sim 11 \%$ more light muscle activity time, but had the same amount of spinal shrinkage compared to office workers using sit workstations during a normal work day. A difference in muscle inactivity of this magnitude is mechanistically linked to acute, clinically significant cardio-metabolic benefits (Dunstan et al. 2012; Duvivier et al. 2013; Pesola et al. 2015), but the benefit of self-reported musculoskeletal discomfort in sit-stand workstation users is unlikely related to spinal shrinkage. These objective crosssectional findings should be confirmed in within-participant designs to provide further evidence on the causal mechanisms of beneficial sit-stand workstation use.

\section{References}

Alkhajah, T. A., Reeves, M. M., Eakin, E. G., Winkler, E. A., Owen, N., \& Healy, G. N. 2012. "Sit-Stand Workstations: A Pilot Intervention to Reduce Office Sitting Time." American Journal of Preventive Medicine 43 (3): 298-303.

Baranowski, T., L. C. Masse, B. Ragan, and G. Welk. 2008. "How many days was that? We're still not sure, but we're asking the question better!" Medicine and Science in Sports and Exercise 40 (7 Suppl): S544-549.

Beynon, C. and Reilly, T. 2001. "Spinal shrinkage during a seated break and standing break during simulated nursing tasks." Applied Ergonomics, 32(6): 617-622.

Chastin, SFM. and Granat, MH. 2010. "Methods for Objective Measure, Quantification and Analysis of Sedentary Behaviour and Inactivity." Gait \& Posture 31 (1): 82-86.

Chau, J. Y., van der Ploeg, H. P., Van Uffelen, J. G., Wong, J., Riphagen, I., Healy, G. N., ... and Brown, W. J. 2010. "Are workplace interventions to reduce sitting effective? A systematic review." Preventive medicine, 51 (5): 352-356.

Chau, J. Y., Daley, M., Dunn, S., Srinivasan, A., Do, A., Bauman, A. E., and van der Ploeg, H. P. 2014. "The Effectiveness of Sit-Stand Workstations for Changing Office Workers' Sitting Time: Results from the Stand@Work Randomized Controlled Trial Pilot." The International Journal of Behavioral Nutrition and Physical Activity 11, 127.

Clemes, S. A., O'Connell, S. E., and Edwardson, C. L. 2014. "Office Workers' Objectively Measured Sedentary Behavior and Physical Activity during and Outside Working Hours." Journal of Occupational and Environmental Medicine / American College of Occupational and Environmental Medicine 56 (3): 298-303.

Dunstan, David W., Bronwyn A. Kingwell, Robyn Larsen, Genevieve N. Healy, Ester Cerin, Marc T. Hamilton, Jonathan E. Shaw, David A. Bertovic, Paul Z. Zimmet, and Jo Salmon. 2012. "Breaking Up Prolonged Sitting Reduces Postprandial Glucose and Insulin Responses." Diabetes Care 35 (5): 976-983.

Dutta, Nirjhar, Gabriel A. Koepp, Steven D. Stovitz, James A. Levine, and Mark A. Pereira. 2014. "Using Sit-Stand Workstations to Decrease Sedentary Time in Office Workers: A 
Randomized Crossover Trial." International Journal of Environmental Research and Public Health 11 (7): 6653-6665.

Duvivier, Bernard MFM, Nicolaas C. Schaper, Michelle A. Bremers, Glenn van Crombrugge, Paul PCA Menheere, Marleen Kars, and Hans HCM Savelberg. 2013. "Minimal Intensity Physical Activity (Standing and Walking) of Longer Duration Improves Insulin Action and Plasma Lipids More than Shorter Periods of Moderate to Vigorous Exercise (Cycling) in Sedentary Subjects when Energy Expenditure is Comparable." PloS One 8 (2): e55542.

Eklund, J. A., \&Corlett, E. N. 1984. "Shrinkage as a measure of the effect of load on the spine." Spine, 9 (2), 189-194.

Finni, T., M. Hu, P. Kettunen, T. Vilavuo, and S. Cheng. 2007. "Measurement of EMG Activity with Textile Electrodes Embedded into Clothing." Physiological Measurement 28 (11): 1405.

Foreman, TK. and Linge, K. 1989. "The Importance of Heel Compression in the Measurement of Diurnal Stature Variation." Applied Ergonomics 20 (4): 299-300.

Hamilton, M. T., Hamilton, D. G., \&Zderic, T. W. 2007. "Role of low energy expenditure and sitting in obesity, metabolic syndrome, type 2 diabetes, and cardiovascular disease." Diabetes, 56(11), 2655-2667.

Haskell, William L., I-Min Lee, Russell R. Pate, Kenneth E. Powell, Steven N. Blair, Barry A. Franklin, Caroline A. Macera, Gregory W. Heath, Paul D. Thompson, and Adrian Bauman. 2007. "Physical Activity and Public Health: Updated Recommendation for Adults from the American College of Sports Medicine and the American Heart Association." Circulation 116 (9): 1081.

Healey, E. L., Fowler, N. E., Burden, A. M., \& McEwan, I. M. 2005. "Repeatability of stature measurements in individuals with and without chronic low-back pain." Ergonomics, 48(11-14), 1613-1622.

Healy, Genevieve, Sheleigh Lawler, Alicia Thorp, M. Neuhaus, E. Robson, N. Owen, and D. Dunstan. 2012. "Reducing Prolonged Sitting in the Workplace (an Evidence Review: Full Report)." Victorian Health Promotion Foundation.

Healy, Genevieve N., Elizabeth G. Eakin, Anthony D. LaMontagne, Neville Owen, Elisabeth A. H. Winkler, Glen Wiesner, Lynn Gunning, et al. 2013. "Reducing Sitting Time in Office Workers: Short-Term Efficacy of a Multicomponent Intervention." Preventive Medicine 57 (1): 43-48.

Igic, I., Ryser, S. and Elfering, A. 2013. "Does Work Stress make You Shorter? an Ambulatory Field Study of Daily Work Stressors, Job Control, and Spinal Shrinkage." Journal of Occupational Health Psychology 18 (4): 469-480.

Ismaila, SO. and Charles-Owaba, OE. 2008. "Determination of the Highest Permissible Spinal Shrinkage." Australian Journal of Basic and Applied Sciences 2 (4): 872-875.

Karakolis, T., \& Callaghan, J. P. 2014. "The Impact of Sit-stand Office Workstations on Worker Discomfort and Productivity: A Review." Applied Ergonomics 45 (3): 799-806.

Kingma, I., van Dieën, J. H., Nicolay, K., Maat, J. J., \&Weinans, H. 2000. "Monitoring water content in deforming intervertebral disc tissue by finite element analysis of MRI data." Magnetic resonance in medicine, 44(4), 650-654.

Leivseth, G. and Drerup. B 1997. "Spinal Shrinkage during Work in a Sitting Posture Compared to Work in a Standing Posture." Clinical Biomechanics 12 (7): 409-418.

Lewis, S. E., \& Fowler, N. E. 2009. "Changes in Intervertebral Disk Dimensions After a Loading Task and the Relationship with Stature Change Measurements." Archives of Physical Medicine and Rehabilitation 90 (10): 1795-1799.

Lis, Angela Maria, Katia M. Black, Hayley Korn, and Margareta Nordin. 2007. "Association between Sitting and Occupational LBP." European Spine Journal 16 (2): 283-298. 
Paul, R. D. and Helander, M. G. 1995. "Effect of Sit-Stand Schedule on Spinal Shrinkage in VDT Operators." Proceedings of the Human Factors and Ergonomics Society Annual Meeting, 39 (10): 563-567, SAGE Publications.

Pesola, A. J., A. Laukkanen, P. Haakana, M. Havu, A. Sääkslahti, S. Sipilä, and T. Finni. 2014. "Muscle Inactivity and Activity Patterns After Sedentary Time-Targeted Randomized Controlled Trial." Medicine and Science in Sports and Exercise 46 (11): 2122-2131.

Pesola, A. J., A. Laukkanen, O. Tikkanen, S. Sipilä, H. Kainulainen, and T. Finni. 2015. "Muscle Inactivity is Adversely Associated with Biomarkers in Physically Active Adults." Medicine and Science in Sports and Exercise 47 (6): 1188-1196.

Pronk, N. P., A. S. Katz, M. Lowry, and J. R. Payfer. 2012. "Reducing Occupational Sitting Time and Improving Worker Health: The Take-a-Stand Project, 2011." Preventing Chronic Disease 9: E154.

Robertson M. M., Ciriello V.M., and Garabet, A.M., 2013. "Office ergonomics training and a sit-stand workstation: Effects on musculoskeletal and visual symptoms and performance of office workers." Applied Ergonomics 44: 73-85.

Rodacki, C. D. L. N., Fowler, N. E., Rodacki, A. L. and Birch, K. 2001. "Repeatability of Measurement in Determining Stature in Sitting and Standing Postures." Ergonomics 44 (12): 1076-1085.

Shrestha, N., Ijaz, S., Kukkonen-Harjula, K. T., Kumar, S., and Nwankwo, C. P. 2014. "Workplace Interventions for Reducing Sitting at Work." The Cochrane Library.

Straker, L., Abbott, R. A., Heiden, M., Mathiassen, S. E., and Toomingas, A. 2013. "Sitstand Desks in Call Centres: Associations of use and Ergonomics Awareness with Sedentary Behavior." Applied Ergonomics 44 (4): 517-522.

Tikkanen, Olli, Piia Haakana, Arto J. Pesola, Keijo Häkkinen, Timo Rantalainen, Marko Havu, Teemu Pullinen, and Taija Finni. 2013. "Muscle Activity and Inactivity Periods during Normal Daily Life." PloS One 8 (1): e52228.

Tikkanen, O., S. Karkkainen, P. Haakana, M. Kallinen, T. Pullinen, and T. Finni. 2014. "EMG, Heart Rate, and Accelerometer as Estimators of Energy Expenditure in Locomotion." Medicine and Science in Sports and Exercise 46 (9): 1831-1839.

Tudor-Locke, C., Schuna, JM., Frensham, LJ. and Proenca, M. 2013. "Changing the Way we Work: Elevating Energy Expenditure with Workstation Alternatives." International Journal of Obesity 38(6): 755-765.

Tuomi, K., J. Ilmarinen, A. Jahkola, L. Katajarinne, and A. Tulkki. 1998. "An Approved Version of the Work Ability Index." Occupational Health series 19, Finnish Institute of Occupational Health, Helsinki.

Van Deursen, L. L., Van Deursen, D. L., Snijders, C. J., and Wilke, H. J. 2005. "Relationship between Everyday Activities and Spinal Shrinkage." Clinical Biomechanics 20 (5): 547550.

Van Dieën, J.H., De Looze, M.P., Hermans, V., 2001. "Effects of dynamic office chairs on trunk kinematics, trunk extensor EMG and spinal shrinkage." Ergonomics 44 (7), 739750.

Van Dieën, J.H. and Oude Vrielink, H.H. 1998. "Evaluation of Work-Rest Schedules with Respect to the Effects of Postural Workload in Standing Work." Ergonomics 41 (12): 1832-1844.

Wilmot, E. G., Edwardson, C. L., Achana, F. A., Davies, M. J., Gorely, T., Gray, L. J., ... and Biddle, S. J. 2012. "Sedentary Time in Adults and the Association with Diabetes, Cardiovascular Disease and Death: Systematic Review and Meta-Analysis." Diabetologia 55 (11): 2895-2905. 


\section{Tables}

Table 1. Demographics and self-reported occupational sedentary time in the two study groups ${ }^{\text {a }}$

\begin{tabular}{lllll}
\hline Characteristics & $\begin{array}{l}\text { Sit-Stand group } \\
(\mathrm{n}=10)\end{array}$ & $\begin{array}{l}\text { Sit group } \\
(\mathrm{n}=14)\end{array}$ & $\begin{array}{l}\text { All } \\
(\mathrm{n}=24)\end{array}$ & $\begin{array}{l}\text { p for group } \\
\text { difference }\end{array}$ \\
\hline Age (years) & $41.0 \pm 11.5$ & $35.3 \pm 9.5$ & $37.7 \pm 10.5$ & 0.150 \\
Height (cm) & $170.2 \pm 6.9$ & $169.8 \pm 7.5$ & $169.9 \pm 7.1$ & 0.882 \\
Body mass (kg) & $67.4 \pm 10.7$ & $69.6 \pm 10.8$ & $68.6 \pm 10.6$ & 0.626 \\
BMI (kg/m $\left.{ }^{2}\right)$ & $23.2 \pm 3.4$ & $24.0 \pm 2.5$ & $23.7 \pm 2.8$ & 0.504 \\
WHR (Waist cm/Hip cm) & $0.81 \pm 0.067$ & $0.86 \pm 0.061$ & $0.84 \pm 0.067$ & 0.081 \\
Proportion of females (\%) & $60.0(6)$ & $57.1(8)$ & $58.3(14)$ & 0.889 \\
Computer use at work (\%) & $90.0(9)$ & $100.0(14)$ & $95.8(23)$ & 0.227 \\
Physical activity (\%) & $70.0(7)$ & $78.6(11)$ & $75.0(18)$ & 0.665 \\
& & & & \\
Occupational sedentary time & $\mathrm{c}$ & & & \\
sitting time (\%) & $62.0 \pm 13.0$ & $83.6 \pm 12.0$ & $74.6 \pm 16.3$ & $\mathbf{0 . 0 0 1}$ \\
standing time (\%) & $36.5 \pm 21.9$ & $18.2 \pm 21.8$ & $25.8 \pm 23.2$ & $\mathbf{0 . 0 3 1}$ \\
computer sitting time (\%) & $68.5 \pm 22.1$ & $87.1 \pm 17.7$ & $79.3 \pm 21.3$ & $\mathbf{0 . 0 2 8}$ \\
computer standing time (\%) & $23.7 \pm 18.0$ & $3.0 \pm 10.7$ & $11.6 \pm 17.3$ & $\mathbf{0 . 0 0 0}$ \\
leisure sitting time (hours) & $3.5 \pm 1.2$ & $3.6 \pm 1.2$ & $3.5 \pm 1.2$ & 0.833 \\
\hline
\end{tabular}

${ }^{\text {a }}$ Values are Mean \pm SD or $\mathrm{n}(\%)$.

${ }^{\mathrm{b}}$ Meets the updated physical activity and health recommendations and participates in at least 30 minutes of moderate-intensity physical activity (which should be accumulated in bouts of at least 10 minutes) on 5 days per week or relatively more intense exercise for less time (20 minutes on 3 days per week) (Haskell et al. 2007, 1081).

${ }^{c}$ Values correspond to self-reported percentage of work time spent sitting and standing, percentage of computer work time spent sitting and standing, and average leisure sitting time in the last three months.

* Bold p values indicate a significant difference between groups. 
Table 2. Workday EMG-derived muscle activity and spinal shrinkage in the two study groups ${ }^{\text {a }}$

\begin{tabular}{|c|c|c|c|c|}
\hline Characteristics & $\begin{array}{l}\text { Sit-Stand group } \\
(\mathrm{n}=10)\end{array}$ & $\begin{array}{l}\text { Sit group } \\
(n=14)\end{array}$ & $\begin{array}{l}\text { All } \\
(n=24)\end{array}$ & $\begin{array}{l}\mathrm{p} \text { for group } \\
\text { difference }\end{array}$ \\
\hline \multicolumn{5}{|l|}{ EMG-derived muscle activity ${ }^{\mathrm{b}}$} \\
\hline Recording time (min) & $420.1 \pm 65.9$ & $392.3 \pm 23.5$ & $403.9 \pm 47.0$ & 0.770 \\
\hline Inactivity time $(\%)$ & $66.2 \pm 17.1$ & $80.9 \pm 6.4$ & $74.8 \pm 13.9$ & 0.014 \\
\hline Activity time (\%) & $33.8 \pm 17.1$ & $19.1 \pm 6.4$ & $25.2 \pm 13.9$ & 0.014 \\
\hline Light activity time $(\%)$ & $26.1 \pm 12.3$ & $14.9 \pm 6.3$ & $19.6 \pm 10.6$ & 0.019 \\
\hline Moderate-to-vigorous activity time (\%) & $7.7 \pm 7.4$ & $4.2 \pm 2.1$ & $5.7 \pm 5.2$ & 0.349 \\
\hline No. of bursts & $601.2 \pm 97.5$ & $563.4 \pm 238.6$ & $579.1 \pm 190.4$ & 0.600 \\
\hline Sum of 5 longest inactivity periods (min) & $41.6 \pm 17.6$ & $60.8 \pm 37.4$ & $52.8 \pm 31.7$ & 0.178 \\
\hline Spinal shrinkage $(\mathrm{mm})^{\mathrm{c}}$ & $5.62 \pm 2.75$ & $6.11 \pm 2.44$ & $5.91 \pm 2.53$ & 0.653 \\
\hline
\end{tabular}

${ }^{\text {a }}$ Values are Mean $\pm \mathrm{SD}$ or $\mathrm{n}(\%)$.

${ }^{\mathrm{b}}$ Muscle inactivity time (time spent on $<90 \% \mathrm{EMG}_{\text {standing), }}$ ), muscle light activity time ( $90 \%$

$\left.\mathrm{EMG}_{\text {standing }}-<\mathrm{EMG}_{\text {walking }}\right)$, muscle moderate-to-vigorous activity time ( $\left.\geq \mathrm{EMG}_{\text {walking }}\right)$.

${ }^{\mathrm{c}}$ Spinal shrinkage between the beginning and end of the workday.

* Bold $\mathrm{p}$ values indicate a significant difference between groups. 


\section{Figures}

Figure 1. Mean EMG amplitudes from the reference tests and thresholds for different activity levels (Mean \pm SD) ${ }^{a}$

${ }^{a}$ Inactivity was defined as EMG amplitude below 90\% of standing EMG and moderate-to-vigorous activity was defined as EMG amplitude above preferred walking speed $(4.1 \pm 0.4 \mathrm{~km} / \mathrm{h})$. These thresholds were determined for each subject individually. Average normalized muscle activity of lying down, sitting, standing and squatting with body weight were $2.2 \pm 1.5 \%, 3.6 \pm 2.6 \%, 19.5 \pm 10.7 \%$ and $287.1 \pm 123.2 \%$ of walking EMG, respectively.

Figure 1

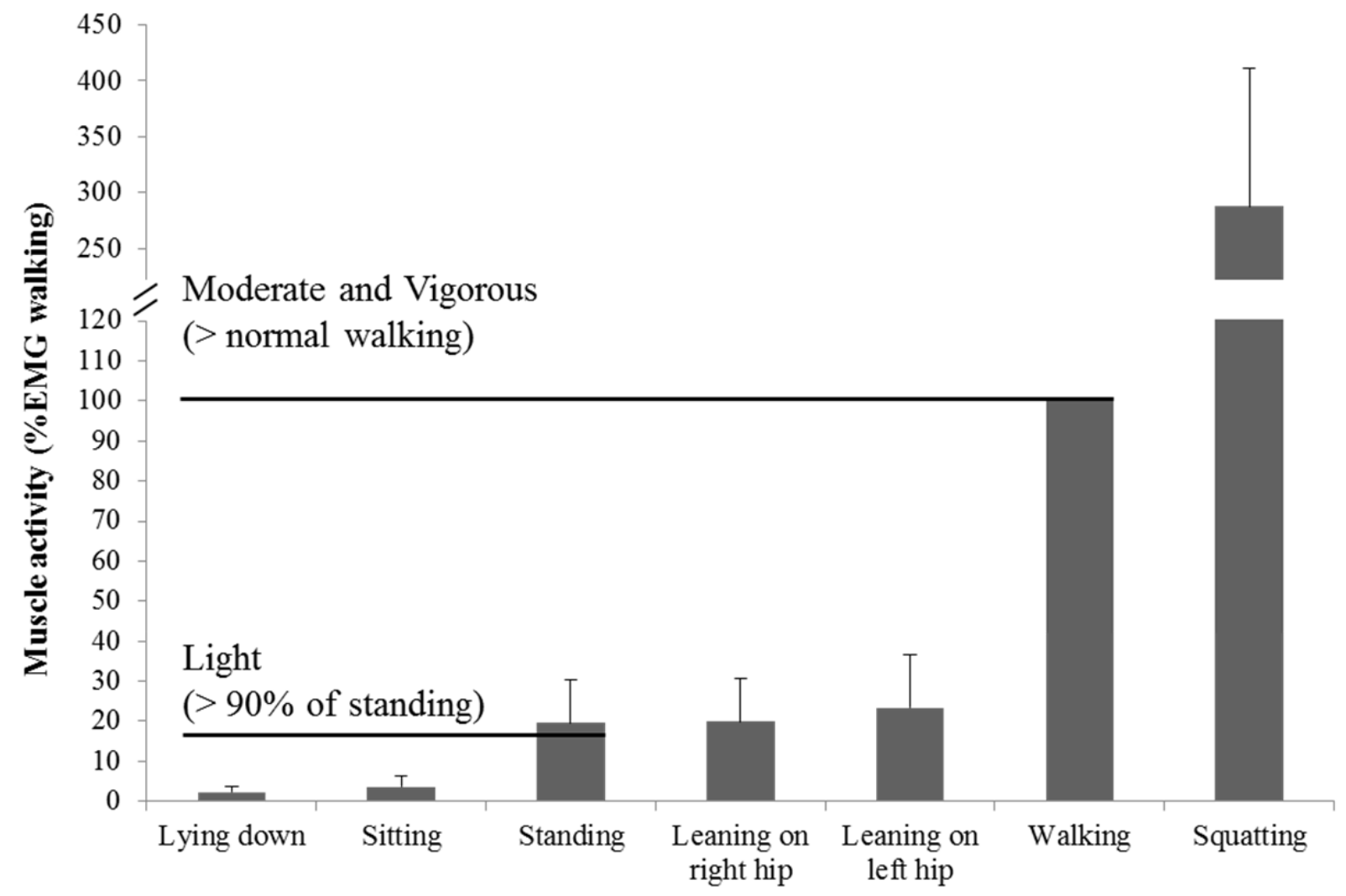

